\title{
Autonomous and Semi-autonomous Radio Commanding of VZLUSAT-1 Nanosatellite from Ground Control Station in Pilsen
}

\author{
Ivo Veřtát, Richard Linhart, Luděk Dudáček \\ Dep. of Applied Electronics and Telecommunication \\ University of West Bohemia \\ Pilsen, Czech Republic \\ ivertat@kae.zcu.cz
}

\author{
Vladimír Dániel, Petr Svoboda \\ Aerospace Research and Test Establishment \\ VZLÚ \\ Prague, Czech Republic \\ daniel@vzlu.cz
}

\begin{abstract}
Nano- and pico- satellites are currently used as low cost in-orbit-demonstrators of new technologies or for the university research. These satellites are launched to the low Earth orbits and radio commanded from the ground control stations. Most of them are equipped with narrow band transceivers working in UHF radio amateur frequency band, reaching only low data transmission rates. Low Earth orbits also limits the number and duration of satellite passing in the range of ground control station. One ground control station has approximately six satellite passes per day, each with duration only in few minutes. This strictly limits the amount of transmitted data between satellite and ground control station. In this paper the software controlled and assisted radio commanding of VZLUSAT-1 nanosatellite during the QB50 scientific mission is described. Two basic modes of satellite radio commanding are described with the aim to increase data throughput during commanding. Software controlled autonomous and software assisted semi-autonomous modes allow effective commanding of satellite while keeping the attention of satellite operator to the satellite condition and keeping the full control over the radio transmission as radio amateur regulation requires. Also the critical operational limits of satellite electronics faces to cosmic vacuum and radiation environment have to be continuously monitored for safety satellite operation.
\end{abstract}

Keywords - satellite commanding; human to machine interface; VZLUSAT-1; QB50; CubeSat; picosatellite; nanosatellite

\section{INTRODUCTION}

VZLUSAT-1 (in fig. 1) is the Czech technology nanosatellite which will be launched on circular low Earth polar orbit in April 2017 as a part of collective mission QB50 [1]. One part of scientific goals is contribute to the Earth thermosphere research with FIPEX sensor unit [2]. The rest of satellites is dedicated for in-orbit-demonstration of several new inhouse technologies.

FIPEX is able to distinguish and measure the timeresolved behavior of atomic and molecular oxygen as a key parameter of the lower thermosphere. Own experiments include the wide-angle X-ray imaging system with Timepix detector [3] based on lobster eye optics, onboard health monitoring of radiation hardened composite materials (mechanical properties measurement by piezo element, volatiles measurement by digital HYT and analog HAL sensor series, radiation shielding effect measurement by XRB diodes) [4], composite hollow retroreflector array for laser ranging and composite substrate for solar panel.

Satellite core is based on GomSpace company products, e.g. NanoPower supply board, NanoMind onboard computer and NanoCom $\mathrm{U} 482 \mathrm{C}$ radio transceiver. Transceiver and onboard computer have functional counter side at the ground control station. It consists of software terminal application CSP-Term based on CubeSat Space protocol (CSP), baseband TNC modem and radio frequency transceiver.

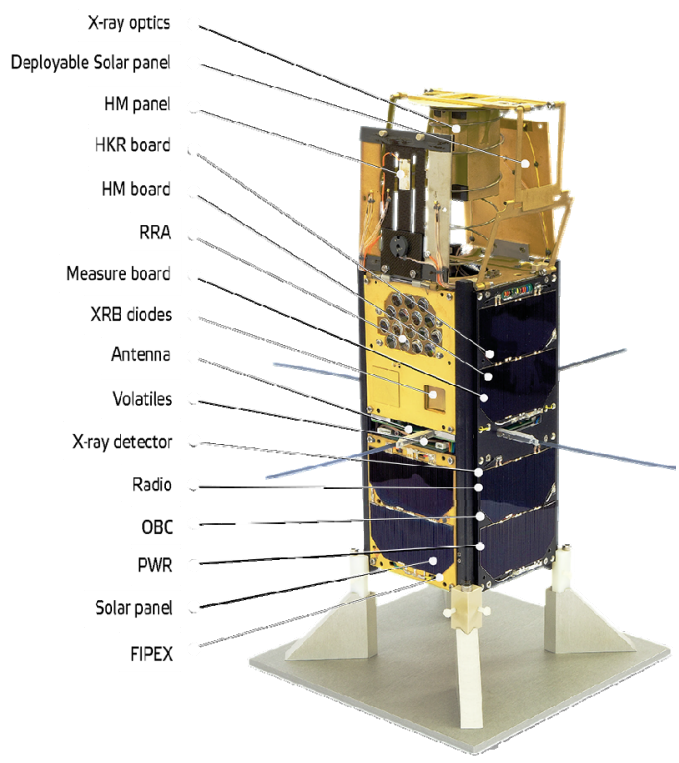

Figure 1. VZLUSAT-1 nanoosatellite.

CSP-Term terminal application is used by satellite operator for typing the commands for satellite and for interpretation of replies and downloaded data. However, such manual commanding of satellite during short satellite pass in range of ground control station do not allow effective control of satellite and managing of data download. During the satellite commanding there is necessary to run many other processes at the same time [5]. For example, the actual satellite position have to be predicted from orbital elements (TLE - two line elements) for ground control 
station directional antenna pointing and for proper Doppler frequency shift compensation on the transceiver. Doppler frequency shift also depends on the direction of communication. It means that the sign of shift is different for download and upload direction of communication. During the commanding the satellite condition have to be monitored [6], because a radio transmission is high power demanding for such small satellite. Temperatures of radio power amplifier as well as Li-Ion accumulator temperature can reach operational limits very fast in cosmic vacuum and also the deep discharging of Li-Ion accumulators is a threat for next proper satellite functionality. Also the satellite operator have to be continuously informed about newly generated data onboard the satellite and state of its download.

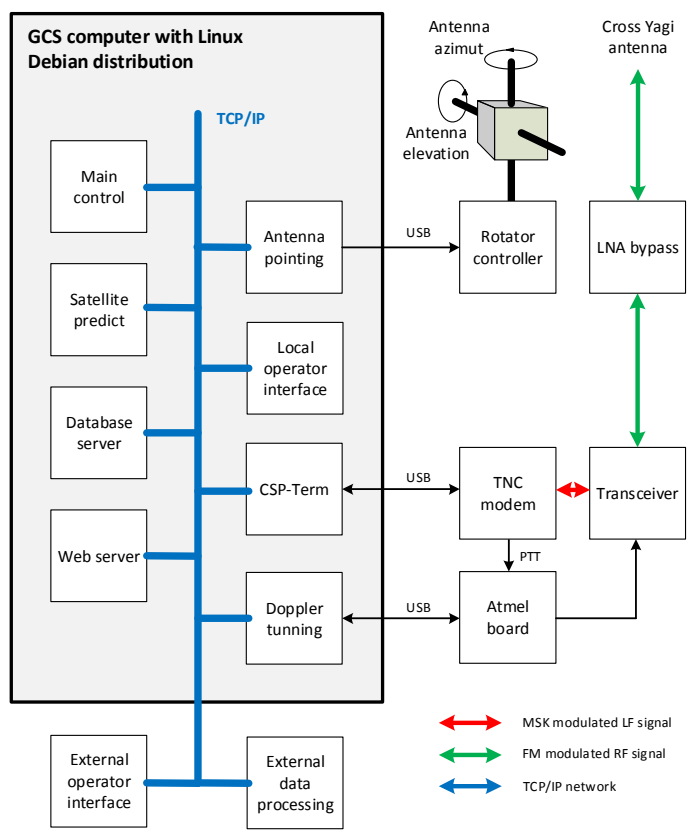

Figure 2. Ground control station infrastructure.

For the effective satellite commanding all the previous tasks have to be automatized, but the possibility of immediately intervention of operators have to be maintain.

The rest of paper is organized as follows. In the section II the basic hardware and software architecture of ground control station is described. Section III describes the main control software for the satellite commanding, including the data handling, processing and checking the critical limits of onboard electronic systems. In the section IV the web based interface for satellite operators and scientific teams is presented. In section V the ground tests of VZLUSAT-1 satellite commanding and operating is briefly summarized.

\section{GROUND CONTROL STATION INFRASTRUCTURE}

The ground control station (GCS) consists of common hardware and software with information signal (commands, data) and control signal (transmit/receive, antenna targeting, Doppler shift tuning) interconnected as shown in fig. 2.

In uplink direction (from GCS to satellite) the commands and data content are generated by CSPTerm software and through USB interface transferred into external TNC modem, where there are packetized, secured by FEC coding and modulated into acoustic signal by MSK modulation. Low frequency MSK modulated signal is fed into ICOM-910H transceiver modulation input, frequency modulated and up converted into UHF frequency band. Transceiver output is directed to the antenna through the controllable rf switch unit. In downlink direction (from satellite to GCS) the received signal from antenna is fed through rf switch unit and low noise preamplifier into transceiver input, down converted and frequency demodulated. Audio output with MSK signal inputs the TNC modem, where the signal is demodulated, FEC checked and depacketized. Replies to commands and downloaded data are sent via USB to the CSPTerm.

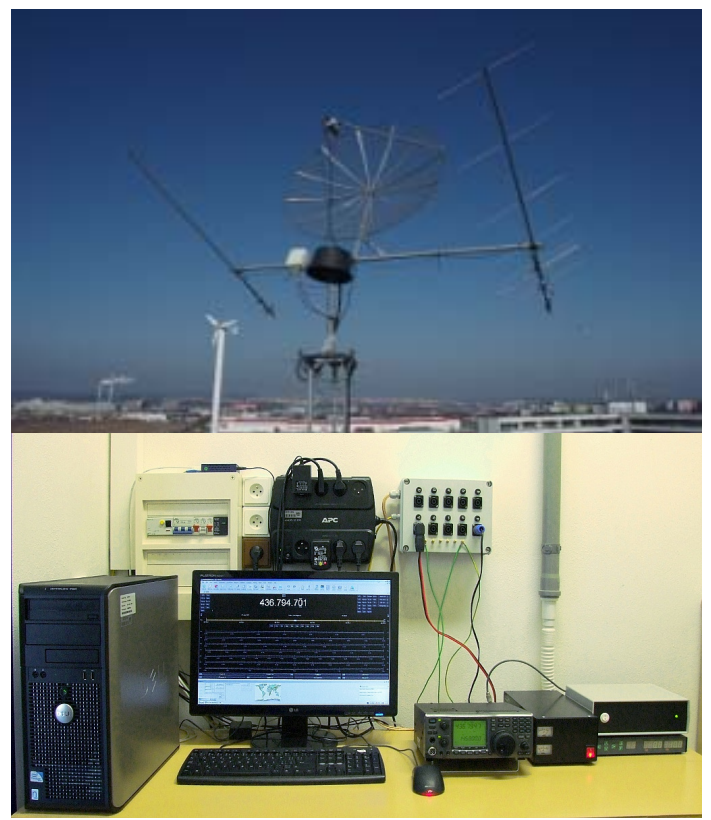

Figure 3. Pilsen ground control station antenna tower and computer control room.

The ground control station (fig. 3) requires several control signals distribution. Antenna rotator get coordinates for proper antenna targeting from GCS computer, based on the satellite position prediction, ground station coordinates and current time. Prediction software also generates Doppler shift compensation signal for Atmel based microcontroller. This microcontroller also gets the PTT (transmit or receive) signal from TNC modem. Microcontroller directly tunes the frequency of transceiver based on PTT signal and Doppler shift compensation signal. Direct connection of Doppler shift compensation signal and PTT signal with transceiver is not possible, because the ICOM-910H transceiver is not capable to store both frequencies (for uplink and downlink) as well as the reading of the PTT signal by GCS computer and sending one proper frequency suffer from high latency. In such case the beginning of transceiver transmission will start with downlink frequency due to latency in reading of the PTT signal by GCS computer and satellite will receive incomplete commands. RF switch placed between antenna and transceiver gets control signal directly from transceiver for bypassing the low noise amplifier during the transmission. 
For the generation of antenna pointing signal and Doppler shift compensation signal the GCS computer run the open source Predict software and use Hamlib libraries. The computer also runs the MySQL database server and Apache web server for storing and sharing of satellite related data. However, the main control software for the satellite commanding through noninteractive mode of CSP-Term and the operator interface to this software is the most interesting and unique part of the ground control station in Pilsen, allowing the autonomous and semi-autonomous operation of satellite.

\section{MAIN CONTROL SOFTWARE}

CSP-Term software allows to use the library of standard and in-house commands for operating the satellite and its subsystems. In common interactive mode the commands are typed by operator in console window. However, in non-interactive mode of CSPTerm its input and output could be redirected to other software.

The main control software is realized in PHP scripting language due to wide support of text string, database and TCP/IP socket communication functions. PHP language could also be effectively used for creating the web based interface between operators, databases and main control software.

The main control software (fig. 4) could run in full autonomous mode (no operator activity is required) or in semi-autonomous mode (some at least minimal operator activity is expected) based on settings in operator interface, as will be explained in section IV. It starts with fixed part of procedures. In the first step the procedure periodically checks the actual satellite position by sending question to the satellite predict application and waits until the satellite cross the coverage boundary of the ground control station and arises to the minimal required elevation for enabling the radio transmission.

In the next steps the ping commands are periodically transmitted until the satellite reply is successfully received. After that, several routines are proceeded, e.g. synchronization of satellite clock, checking the basic satellite status (temperatures, voltages of accumulators, number of resets) and downloading the datakeeper preview. The satellite operator is announced about exceeding of nominal operational limits and can make changes in optional part of commanding procedure or also can disable the autonomous mode of commanding and solve the critical problem by manual commanding through interactive mode of CSP-Term.

Operator dependent part of control script start with new checking of actual satellite condition after fixed part of script and the next steps are done or not based on reading the database with stored requirements of satellite operators or requirements generated by autonomous preprogrammed logic. This covers planner upload (file with tasks scheduler for satellite onboard computer), arbitrary command execution, data upload and data download. These steps are periodically proceeded until the satellite is above the horizon, there are still some unexecuted tasks and satellite condition is in limits.

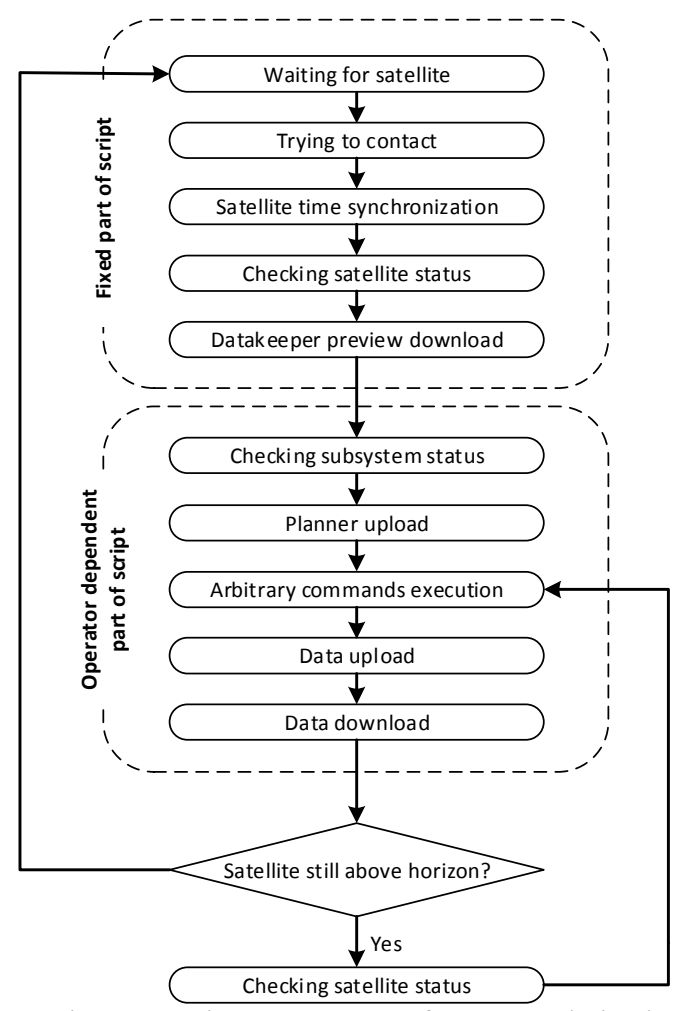

Figure 4. Time sequence of commands in the main control software during the satellite commanding.

The semi-autonomous mode require some decision of satellite operators, at least what data sections should be downloaded and if a new planner should be uploaded. Operator also can place arbitrary commands into the queue to transmit. On the other hand, the full autonomous mode do not require decisions of satellite operators. Requirements to data download and some arbitrary commands to transmit are generated by preprogramed logic (external data processing unit, see fig. 2), which is based on data stored in database (especially on downloaded datakeeper preview, operator configuration and history of commands execution).

The datakeeper preview is a very important information received from satellite. It contains how many data segments (labeled as chunks) of the onboard satellite memory (divided into particular subsystem storages) were recorded by particular satellite subsystems and datum of the last record for each storages. Based on this downloaded datakeeper preview, history and results of executed commands, the external data processing unit generates the preview of state of chunks for each storage. The state of chunks could be one of follows:

- chunks transfer not planned

- chunks transfer planned, but not started

- chunks transfer attempted, it will be repeated

- chunks transfer failed, no more attempts

- chunks transfer successful

- chunks planned to delete in satellite memory

- chunks deleted in satellite memory 
The state of the storage chunks is presented to satellite operators and in semi-autonomous mode they can decide what should be downloaded or deleted by generating the request for downloading or deleting. In full autonomous mode these requests are generated by external data processing unit.

\section{WEB BASED INTERFACE FOR OPERATORS}

Operating and commanding of satellite on the low Earth orbit is time demanding task, because the satellite is passing above ground control station approximately six times per day (it depends on orbit altitude and ground control station latitude) with approximately 90 minutes minimal distances between two satellite passes. It requires to keep at least two satellite operators take turns in shifts permanently 24 hour a day. The possibility to operate the ground control station remotely via TCP/IP network from home or business trip can take the strain off from operators, at least in late evening or early morning times. For this purpose it was proposed the web based interface between satellite operators, main control software and database.

Web based interface for the satellite operators and members of scientific teams have to allow easy control over the satellite commanding, supervision of the satellite critical parameters (subsystem temperatures, accumulator voltage and current, power bus currents, onboard computer workload, number of resets) and accessing the downloaded scientific data. For interface clarity the data, forms and control button are grouped into several tabs, each with particular function and purpose. Full screen mode of web browsers allow to display two arbitrary tabs side-by-side on full HD screens (1920x1080 resolution) as is shown in fig. 7 or four arbitrary tabs (matrix of $2 \times 2$ tabs) on ultra HD screens ( $3840 \times 2160$ resolution). Therefor the satellite operators could see all necessary information for satellite commanding on one screen. The most important tabs will be described in the next subchapters.

\section{A. Pass info tab}

It contain the basic information about previous and future passes of satellite above two ground control stations (mother and cooperating ground station). This tab allow a better planning of operator work shifts and planning the cooperation with other ground control station. Each pass info record contains information about pass beginning time (AoS), pass duration, maximal elevation during pass and two line element version used for prediction of pass. Satellite pass prediction is made for at least next 2 days and it is periodically updates with each new two line element version.

\section{B. Comm info tab}

It is the main tab showing the actual state of radio communication with satellite and actual obtained state of the satellite. It helps to keep overview over the satellite status and to make easier decisions for operators. It contains the communication state window with the state of main control software procedures, antenna window with refreshed images of antenna tower with 2-axis rotator, last received WOD and OBC windows with the satellite critical parameters (temperatures, currents, voltage,...), actual pass window with basic information about current pass, datakeeper window with the last received datakeeper preview, text console window with details of actual executed commands and windows with list of next several download requests, upload requests and arbitrary commands in queue.

\section{Stor info tab}

It is the second most important tab for operators and it contains the information about data chunks status for all storages and several control buttons. Each storages belongs to particular satellite subsystems or to particular function. The color of data chunks shows one of seven possible state of chunks (see section III). Buttons under chunks state allow to switch into full autonomous mode, in which the requests to data download of still not downloaded data are generated automatically as well as commands to chunks deletion in satellite memory after successful download.

\section{DK req tab}

This tab allows manual adding of requests for data chunks downloading and files uploading in semiautonomous mode. Satellite operators also can give the priority for each download request, set the lossless compression during data downloading and temporally suppress the execution of such download request.

\section{E. CMD req tab}

This tab allows manual adding of the arbitrary commands to the queue for execution. Satellite operators can give the priority for each command, temporally suppress the execution of command and set the delay before executing next commands. Satellite operators are able to view the satellite response to each executed commands.

\section{F. $\quad O B C$ WOD $t a b$}

This tab shows the long term continuously course of whole orbit data set, which contains all important information about satellite status and health, periodically sampled as it is required by QB50 mission headquarters. This data set is usually downloaded automatically by main control software (but with the option to deactivate it) and data files are transferred to QB50 headquarters. Satellite operators can monitor a long term trends in current drains, accumulator voltage cycling, subsystems overheating and QB50 scientific unit status.

\section{G. RT data tab}

This tab shows the short term course of OBC housekeeping data a whole orbit data during the actual radio commanding session. Data are periodically checked by the main control software as a prevention of overheating satellite radio unit and accumulators or accumulators over discharging.

Other tabs contain less important data for satellite operators. The visualization of satellite flying above the Earth fulfils also important public relation issue 
and can motivate a young students to space hardware design and experiments. Direct copy of TNC console window also could be interested for technical enthusiastic people for the deep insight of satellite commanding. The last tab contains the downloaded and decoded data files for VZLUSAT-1 scientific teams.

\section{GROUND TEST OF SATELLITE COMMANDING}

During 2015 and 2016 there were realized several ground radio commanding tests with VZLUSAT-1, placed outside the laboratory environment. These tests were done for short ( $\sim 9 \mathrm{~km}$, Pilsen to Radyne hill) and middle ( $26 \mathrm{~km}$, Pilsen to Brno hill) communication distances.
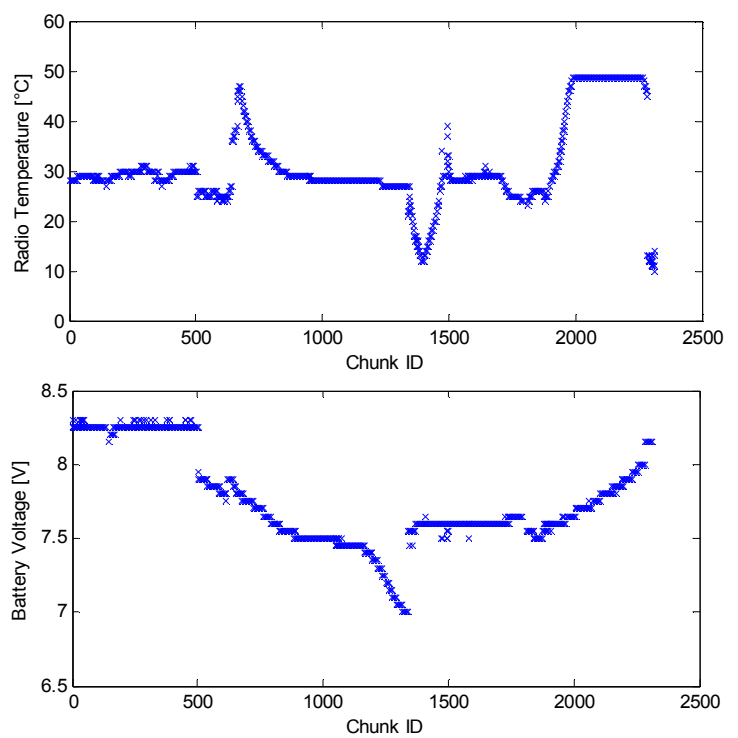

Figure 5. Sample of downloaded WOD data sets during the ground commanding tests.

Satellite commanding through web interface were successfully verified, including automatic downloads of whole orbit data chunks, upload of planner script files, execution of arbitrary commands and monitoring of satellite health status.

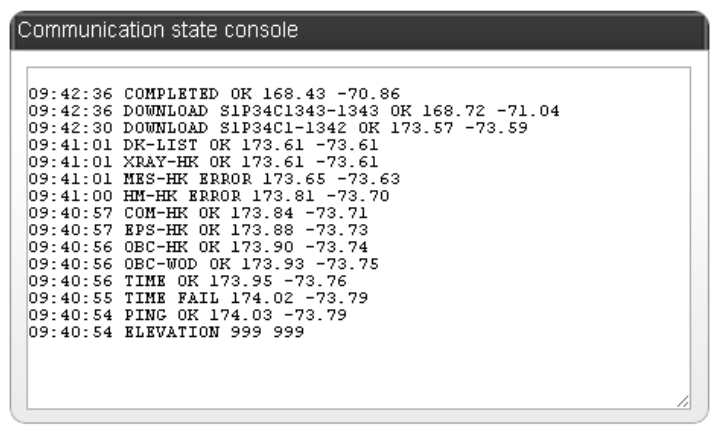

Figure 6. Simplified log from the main control software, periodically updated on the web based operator interface.

Samples of downloaded data are presented in fig. 5. Battery voltage and radio subsystem temperature shows a short history of satellite testing. For example, since chunk ID approximately 1900 it starts preparation to cycling test (thermal cycling during vacuum condition) in thermo-vacuum chamber to verify the functionality in operation temperature limits. Fig. 6 shows the log information during radio commanding test. Some subsystems report errors, because the satellite was equipped only with $\mathrm{COM}$ (radio), EPS (power supply) and OBC (onboard computer) subsystems in the time of this radio session.

\section{RESULTS AND CONCLUSIONS}

Ground control station in Pilsen was equipped with hardware and software solution for long-term operating of VZLUSAT-1 nanosatellite during international scientific collaborative mission QB50. The workload for satellite operators and mission headquarter was minimized by software control and software assistance of many tasks. Antenna pointing toward the satellite position and Doppler frequency shift is controlled automatically based on satellite pass predictor. The in-house electronic board with simple Atmel controller was designed for fast switching between uplink and downlink frequency shift compensation without lag of PC's operating system. This reduces the necessity of long preamble placed before transmitted data and increases the packet throughput. However, our most important contribution is the main control software linked up to web based operator interface. It enables to keep the full control of operators over the satellite commanding as well as full automation of the most procedures (satellite health checking, onboard time synchronization, data download planning, etc.) if required. VZLUSAT-1 is planned to launch at the end of April 2017 from India space center, together with other seven satellites from the third batch of QB50.

\section{ACKNOWLEDGMENT}

This project was funded by TA ČR under project number TA04011295: Wide-angle X-ray imaging system with Timepix detector. This work was also supported by SGS-2015-002, inner project for student activities at University of West Bohemia.

\section{REFERENCES}

[1] E. Gill, P. Sundaramoorthy, J. Bouwmeester, B. Zandbergen, R. Reinhard, "Formation flying within a constellation of nano-satellites: The QB50 mission,“ in Acta Astronautica, Volume 82, Issue 1, January 2013, Pages 110-117, ISSN 0094-5765.

[2] P. Roßmans, "OB50 FIPEX Science Unit Interface Control Document. Issue 2. “, Technical report, TU Dresden, 2014.

[3] T. Baca, M. Platkevic, J. Jakubek, A. Inneman, V. Stehlikova, M. Urban, O. Nentvich, M. Blazek, R. McEntaffer, V. Daniel, " Miniaturized X-ray telescope for VZLUSAT-1 nanosatellite with Timepix detector," Journal of Instrumentation, Volume 11, 2016, ISSN: 1748-0221

[4] V. Dániel, M. Urban, V. Stehlíková, O. Nentvich, "VZLUSAT-1: verification of new materials and technologies for space." In SPIE Optical Engineering+ Applications (pp. 99780N-99780N). International Society for Optics and Photonics. 2016.

[5] M. Bazdresch, S. Velayudhan and W. Johnson, "A satellite ground station for teaching digital and wireless communications," 2016 IEEE Frontiers in Education Conference (FIE), Eire, PA, 2016, pp. 1-4. doi: 10.1109/FIE.2016.7757672

[6] D. A. M. Osman and S. W. A. Mohamed, "Hardware and software design of Onboard Computer of ISRASAT1 CubeSat," 2017 International Conference on Communication, Control, Computing and Electronics Engineering (ICCCCEE), Khartoum, Sudan, 2017, pp. 1-4. doi: 10.1109/ICCCCEE.2017.7867654 


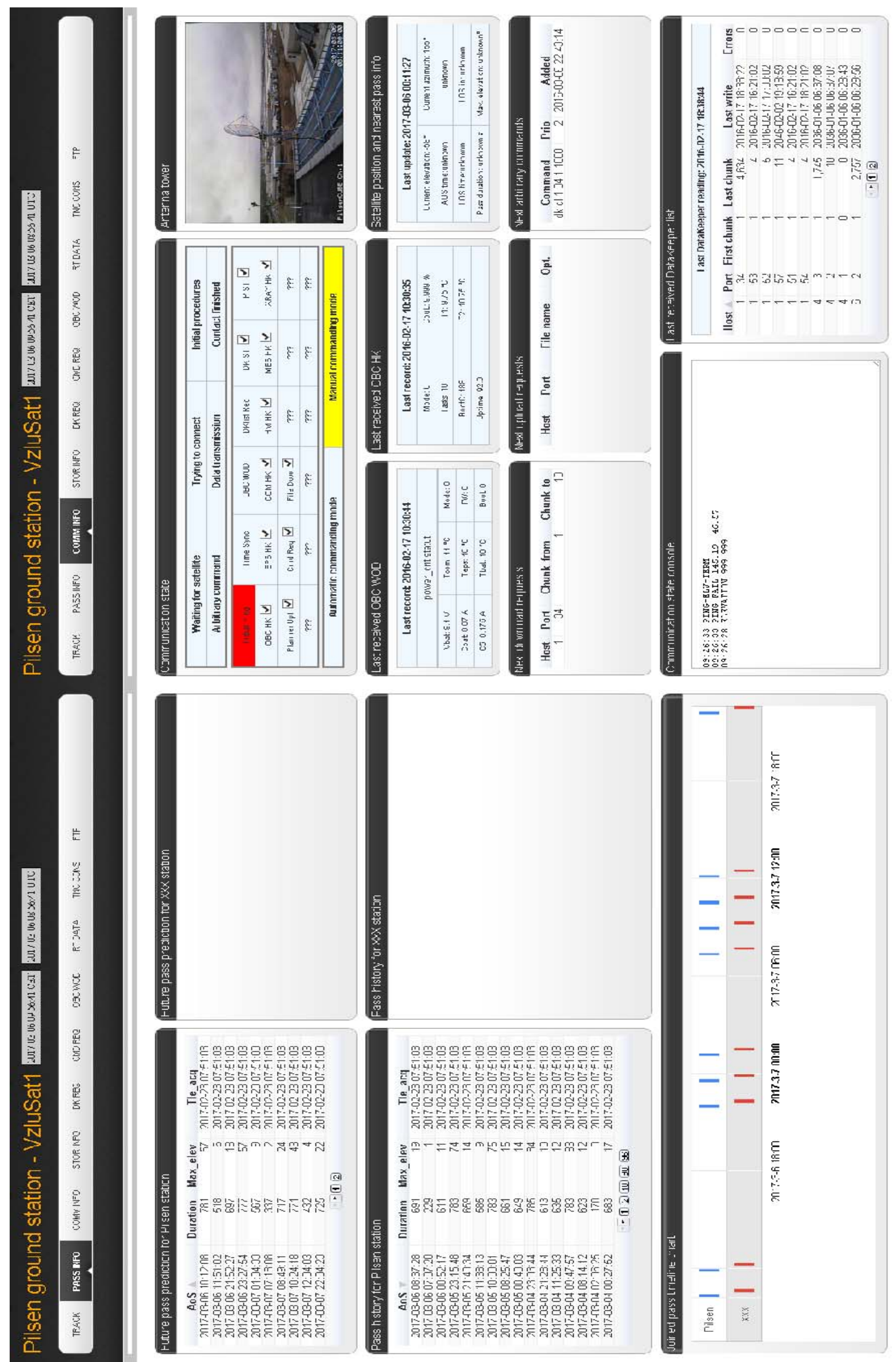

Figure 7. Web based interface for VZLUSAT-1 operators and members of scientific teams. 